\title{
Ciro Scotto / Kenneth Smith / John Brackett (Hg.), The Routledge Companion to Popular Music Analysis. Expanding Approaches, New York: Routledge 2019
}

Schlagworte/Keywords: Analyse von populärer Musik; Analysemethoden; media reflexivity; Medienreflexivität; methods of analysis; popular music analysis; Popular Music Studies; study of popular music

Die Analyse populärer Musik erfreut sich einer internationalen Konjunktur und zwar einer, die weit über die formalisierte akademische Arbeit hinausgeht. Mehrere hochwertige YouTubeKanäle bzw. -Playlisten (ShaunTrack, Rick Beato - What Makes This Song Great?, Genius Deconstructed) und Podcasts (Dissect, Switched On Pop oder SongExploder) bieten seit einigen Jahren ausführliche musikalische Analysen von Songs, Tracks und Alben an, die ein breites Publikum adressieren. ${ }^{1}$ Insofern ist den Autoren der kürzlich erschienenen Monographie Switched On Pop. How Popular Music Works, and Why it Matters - die auf einen Podcast zurückgeht - zuzustimmen, wenn sie von »a golden age of popular music analysis « ${ }^{2}$ sprechen. Der hier rezensierte Band setzt sich allerdings von diesen spopulären ‘ Formaten und zugleich von vielen akademischen Publikationen programmatisch ab, die einen Fokus auf bestimmte musikalische Dimensionen, Genres, Künstler*innen oder Songs legen. ${ }^{3}$ Die Herausgeber des Bandes erheben nämlich den ambitionierten Anspruch, aus Theorien und analytischen Instrumenten zur Erforschung zeitgenössischer Kunstmusik (contemporary art music) Ansätze zur Analyse populärer Musik zu gewinnen, ja, sogar neue Paradigmen zu entwickeln. Im Vorwort wird diese anvisierte Er-

1 Die bereits seit Jahren laufende Radiosendereihe Die Musikanalyse von Hartmut Fladt bei RadioEins kann in diesem Zusammenhang als deutschsprachiger Pionier dieser Art musiktheoretischer Vermittlung erachtet werden.

2 Sloan/Harding 2020, 4.

3 Vgl. etwa Fink/Latour/Wallmark 2018; BrøvigHanssen/Danielsen 2016; Chaker/Schermann/ Urbanek 2018; Osborn 2016; Appen/Doehring/ Helms/Moore 2015. weiterung bestimmt "as any compositional, analytical, theoretical, aesthetic, or cultural concept that goes further than current scholarship towards our understanding of the pitchclass structures, form, timbre, rhythm, aesthetics, or cultural significance of various forms of popular music." (xvii)

Der Band ist in fünf Abschnitte gegliedert: (1) »Establishing and Expanding Analytical Frameworks", (2) »Technology and Timbre«, (3) »Rhythm, Pitch, and Harmony", (4)»Form and Structure " und (5) „Critical Frameworks: Analytical, Formal, Structural, and Political«. Im Rahmen einer Rezension ist eine umfassende Inhaltsangabe oder gar eine eingehende kritische Auseinandersetzung mit dem kompletten Band nicht realisierbar oder erstrebenswert. Ziel dieser Rezension ist also vielmehr, bemerkenswerte Beiträge detailliert zu besprechen, ihren Ertrag für eine Erweiterung analytischer Ansätze einzuschätzen und damit letztlich zu beurteilen, ob der Sammelband den selbst erhobenen Anforderungen entspricht. Dabei möchten wir einen Schwerpunkt auf den ersten Abschnitt des Bandes setzen, da dieser zum Ziel hat, analytische Ansätze einzuführen und weiterzudenken. Ferner greifen wir exemplarisch Beiträge aus den anderen Abschnitten heraus, die uns auf ihre je eigene Art und Weise kritikwürdig erscheinen.

\section{ZUR ETABLIERUNG UND ERWEITERUNG MUSIKANALYTISCHER ANSÄTZE}

Beim ersten Abschnitt fällt zunächst auf, dass mit der Ausnahme von Lori Burns und Allan F. Moore keine andere prominente Figur aus den Formationsjahren der Popular Music Studies (PMS) vertreten ist. Das ist insofern bedauer- 
lich, als viele von ihnen wie etwa David Brackett, Richard Middleton, Philipp Tagg oder Sheila Whiteley die musikalische Analyse als zentrales Moment der Erforschung populärer Musik erachtet haben - ein Ansatz, der innerhalb der International Association for the Study of Popular Music (IASPM) längst keine Selbstverständlichkeit mehr ist. ${ }^{4}$ Im ersten Beitrag des Abschnittes "Some Practical Issues in the Aesthetic Analysis of Popular Music « (3-14) bietet Christopher Doll eine pragmatische Orientierung für das an, was er ohne Begriffsbestimmung 'ästhetische Analyser nennt. Nachdem Doll das smusikalische Werkı als Hauptgegenstand jener Analyse bestimmt, behandelt er spezifische methodische Schwierigkeiten der Transkription in Hinblick auf straditionelles Parameter der Musik wie Harmonie, Melodie und Rhythmus. Anhand von konkreten Stücken weist Doll zudem auf Kurzschlüsse, Verkennungen und Irrtümer hin, die sich bei der Verabsolutierung von aus der Kunstmusik stammenden analytischen 'Standards` ergeben. Diese seien somit als ,falscher zu bezeichnen, kämen aber zwangsläufig zur Anwendung, wenn man sie nicht kritisch reflektiere (10). So sinnvoll diese Problematisierung ist, so fraglich ist Dolls Bestimmung der Motivation und des Sinns ,ästhetischer Analyse`:

[...] aesthetic analysis is by its very nature, in its entirety, an activity in pursuit of meaning. It is meaningful to identify a song as a musical work, to transcribe its pitches and rhythms, to consider its relationship with diatonic conventions, to examine its internal and external relationships tonal and textual alike [...]. (12)

Zu der Frage, worin die `Bedeutung, gründet, verweist Doll auf die Überzeugungskraft und 'Leidenschaft der Analytiker*innen: "the ultimate job of analysis is to convince those around us that the meanings we find are illuminating, stimulating, and reflective of our underlying passion for the music (ebd.).

Einen Höhepunkt erreicht der Abschnitt mit den Beiträgen von Phil Ford "Style as Analysis" (15-28) und Giles Hooper »Thank You for the Music« (29-44). Fords Beitrag beginnt mit einer

4 Die Gründung des Network for the Inclusion of Music in Music Studies (NIMiMS) kann als Symptom dafür angesehen werden. kurzen und pointierten fachgeschichtlichen Darstellung des Konflikts zwischen Musikwissenschaftler*innen und spop studies scholars wir möchten beide idealtypischen 'Lager mit den von Tagg vorgeschlagenen Begriffen $>M u-$ sos` und 'Non-Musos` bezeichnen. ${ }^{5}$ Ford zieht , Landkarte und , Gebiet` als Analogien für Notentext und phänomenales Hörerlebnis von Musik heran, um die Probleme fassbar zu machen, die einige Musos bei der Verwechslung beider Instanzen erzeugen. Diese Probleme werden insofern fachpolitisch zugespitzt, als Ford regelrecht performativ einen `Zaubertrickı ankündigt: »Here, I am about to do some magic. Watch « (18) und anschließend Beethovens Appassionata kurz analysiert. Damit zeigt Ford mit humorvollem Scharfsinn, wie sich die Profilierung des musikanalytischen Zugangs durch sein für Non-Musos unverständliches Fachvokabular rechtfertige: "that gives musicology its reputation as the sparticle physics of the humanities.« (ebd.) Die anschließende Kritik richtet sich an die Sachlichkeit und Objektivität, die viele Musos, so Ford, durch die methodische Verdrängung der eigenen Position in ihren Analysen inszenieren. Dagegen fordert er eine explizite Darlegung der eigenen Hörerlebnisse, was an den von einigen Kolleg*innen vorgebrachten sreflexive turn bzw. ssubjective turn erinnert. ${ }^{6}$ In diesem Sinne meint Ford mit ,Analyse als Stil eine individuelle Verortung

5 »I use muso (without the non-) colloquially to denote someone who devotes a lot of time and energy to making music or talking about it, especially its technical aspects. A muso is in other words someone with either formal training in music, or who makes music on a professional or semi-professional basis, or who just sees him/herself as a musician or musicologist rather than as a sociologist or cultural studies scholar. Non-musos are simply those who don't exhibit the traits just described [...].« (Tagg 2015, 3)

6 Vgl. Butler 2006, 16 f.; Doehring 2012, 36 f. Dies scheint Parallelen zu Alexander Rehdings Festellung zu haben, wonach »[p]racticing music theory today, then, seems to mean primarily to theorize the mutual relationship between music and the listener « (2006, 208). Es liegen allerdings analytische Ansätze in den PMS vor, die ohne Bezug auf diese Diskurse eine methodische Reflexion der Analytiker*innen und deren Hörerlebnis leisten, vgl. Müller 2018. 
der Analytiker*innen, die auf ihrem persönlichen Stil und der Darlegung individueller Hörerlebnisse bei der Ausrichtung musikalischer Analysen beruht.

Fords Kritik ist zwar nachvollziehbar und in einigen Punkten zutreffend, allerdings spielt er Objektivität und Subjektivität gegeneinander so aus, dass er ihre dialektische bzw. hermeneutische Vermittlung völlig ausschließt. ${ }^{7}$ So gilt für Ford ein intensiver Dialog zwischen Musikkritik (criticism) und akademischer musikalischer Analyse als einziger Weg zur Überwindung der monierten Objektivierung. Ford gibt keine Gründe hierfür an und verweist lediglich auf persönliche Erfahrungen und die Intuition, dass beide Praktiken ohnehin dasselbe wollen und erreichen. Denn er ist der Überzeugung, dass Leser*innen mit Analytiker*innen größtenteils übereinstimmen, wenn Letztere eine subjektive sdichte Beschreibung، von Musik vorlegen. Dies ist nicht nur eine recht optimistische, ja nahezu naive Einschätzung der intersubjektiven Verfasstheit musikanalytischen Wissens, sondern auch die Offenlegung eines ungewöhnlichen Begriffs von (Musik-)Wissenschaft: »My moment of inner agreement was also a moment of gratitude for being understood. In such moments, you feel a little less alone, and this is all I can ask of criticism. Or scholarship, if I'm being real.« (24) Ob der Sinn wissenschaftlicher Auseinandersetzung (mit Musik) daraus hervorgeht, ja überhaupt damit zusammenhängt, darf bezweifelt werden. Ford kommt jedenfalls seiner Forderung nach Selbstreflexion und eigenem Stil in seinem höchst kreativen und suggestiven Beitrag konsequent nach.

Hoopers Beitrag ist eine fulminante und gut informierte Metakritik der methodologischen Spaltung zwischen der (musikwissenschaftlichen) study of popular music und den (interdisziplinären) PMS. Der Autor kritisiert vor allem die Vernachlässigung >der Musikı in den PMS, die sie gleichsam zur 'Blackbox` gemacht habe. ${ }^{8}$ Man kann auch hier wieder die Begriffe Musos und Non-Musos bemühen. Für die folgende Diskussion zieht Hooper zwei für ihn

$7 \quad$ Eine derartige Vermittlung ist etwa in der philosophischen Ästhetik von Georg W. Bertram (2014, 131-139) ausbuchstabiert worden.

8 Zur Metapher von populärer Musik als >Blackbox`vgl. Appen/Doehring 2014, 219. repräsentative Publikationen aus beiden 'Lagern methodologischen Positionen darzustellen. Dabei stellt der Autor mit gewisser Polemik fest, dass Interdisziplinarität in den PMS kaum realisiert sei. Diese werde vielmehr angenommen, weil die PMS sich aus verschiedenen Disziplinen bzw. Feldern zusammensetzt, doch in der Regel Non-Musos dort weiterhin disziplinär forschen. So gesehen, sei inkonsequent, Musos Konservativismus vorzuwerfen, weil sie snurı musikwissenschaftlich forschten. Zentral für die Argumentation des Beitrages ist jedenfalls, dass die kritisierte methodologische Spaltung auf einer Text-Kontext-Dichotomie gründet (31). Dass diese Spaltung, die auch institutionell und fachpolitisch erkennbar sei, überwunden werden solle, wird in Anlehnung an Kants prominenten Satz in der Kritik der reinen Vernunft auf den Punkt gebracht: »To paraphrase a certain philosopher, stext without context is blind, context without text is empty." " (34)

Anders als Ford stellt Hooper die Musikwissenschaft recht positiv dar - vielleicht viel zu positiv. Der Autor behauptet nämlich, diese sei unter dem Einfluss der New Musicology, des Critical Turn usw. seit langem erfolgreich dabei, Texte und Kontexte zu untersuchen, während die Non-Musos der PMS ausschließlich Kontexte erforschen könnten. So scharf und polemisch Hoopers Einschätzung ist, so ungewiss ist es, ob die meisten Musos tatsächlich hinreichendes Fachwissen besitzen, um Text und Kontext so zu vermitteln, dass Non-Musos dabei wenig auszusetzen hätten. Allerdings besteht in der (Historischen) Musikwissenschaft gewiss eine gründliche Reflexion über die Schwierigkeit einer konzeptuellen und methodischen Vermittlung von Analyse und Geschichte $^{9}$ - eine Reflexion, die in der Analyse populärer Musik grundsätzlich fehlt. AnschlieBend diskutiert der Autor einen Nervenpunkt musikalischer Analyse: die Beziehung zwischen Text und Rezeption. Er behauptet, die Rezeption von Musik sei zumindest teilweise auf die Verfasstheit von Texten bezogen, und schließt daraus, dass es womöglich so viele Eroicas wie Hörer*innen geben könne, aber dass viele Rezeptionen bzw. Interpretationen

9 Vgl. Janz 2012. 
der Eroica ungültig oder falsch sind, weil sie sich nicht auf den Text zurückführen lassen. Der Text ermöglicht und verunmöglicht also bestimmte Rezeptionsweisen - der Begriff affordance wird hier strategisch eingesetzt. ${ }^{10}$ Wird aber Musik analysiert, um zu zeigen, welche Interpretationen gar nicht in Frage kommen?

Es ist zwar klar, dass der Solipsismus und ein radikaler Subjektivismus zu vermeiden sind, aber Analytiker*innen, die Text und Kontext versöhnen wollen, haben zu begründen, warum diese oder jene analytischen Entscheidungen und Interpretationen sinnvoll sind. Und diese Begründung bedarf historischen Wissens über die Musikpraxis, aus der das Musikstück stammt - was nicht nur für populäre Musik mit dynamischen und prägenden Vermittlungsprozessen durch Kulturen und Medien zusammenhängt. ${ }^{11}$ Man kann sich natürlich, wie Hooper nahelegt, mit der pragmatischen Annahme abfinden, dass jede*r irgendwie Musik so hört wie die Analytiker*innen (32). Damit erspart man sich die langwierige Erforschung von Vermittlungsprozessen. Würde aber diese pragmatische Annahme nicht zu einer zirkulären Argumentation führen? Und zwar etwa, wenn man Stücke analysiert, in denen man die 'Materialisierung، postkolonialer, neoliberaler oder patriarchalischer Strukturen hört, weil man davon ausgeht, jede*r hört das so; oder apodiktisch: weil man es so zu hören hat? Würde man da nicht zudem scherry pickings betreiben, wie es viele Musikethnolog*innen gegen kulturkritisch ambitionierte Analysen populärer Musik einwenden? Hooper geht auf solche Schwierigkeiten nicht ein.

Damit ist aber diese schlaglichtartige Metakritik noch nicht zu Ende. Hooper stellt der in den PMS mittlerweile dominierenden Musikethnologie eine polemische wie wichtige Frage: Worin liegt ihre Musik- oder Klangspezifik?

10 Allan F. Moore hat als erster diesen aus der Psychologie stammenden Begriff zur Analyse populärer Musik herangezogen (1993, 6 f.). Man denke auch an Peter Wickes medialen Musikbegriff (1992, 30-39), der Ähnliches leistet.

11 Antoine Hennion hat diesen Punkt exemplarisch an der Rezeption von J. S. Bach gezeigt (2015, 247-260)
Diese Frage ist insofern brisant, "since exactly the same approach could have been adopted, and in fact has been adopted, in investigating the behaviour and rituals of grocery-shoppers in supermarkets." (33) In Kontrast dazu wird am Beispiel eines analytischen Musos-Ansatzes die fundamentale Frage nach der pertinence bzw. Angemessenheit aufgeworfen. Hooper behauptet, die Analytiker*innen verfehlten offenkundig den Sinn von Blues, wenn sie funktionsharmonisch analysierten. (ebd.) Dennoch sei es, so räumt Hooper später ein, ein Fehlschluss zu glauben, "that it is inappropriate to refer to smodal subversion Nirvana's 'Heart Shaped Box supertonic when describing Lionel Richie's >All Night Long، simply because the listeners (in whichever cultural context) would not conceptualise the music in those terms [...].« (37) Hooper bestimmt zwar Ursachen für das Problem der Unangemessenheit, begründet Angemessenheit aber nicht: ${ }^{12}$

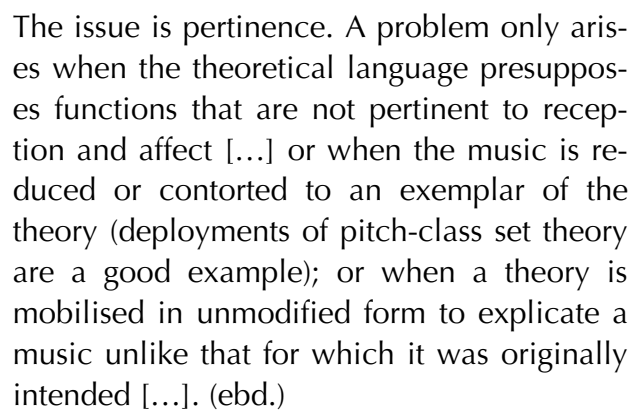
es when the theoretical language presupposes functions that are not pertinent to reception and affect $[\ldots]$ or when the music is reduced or contorted to an exemplar of the theory (deployments of pitch-class set theory are a good example); or when a theory is mobilised in unmodified form to explicate a music unlike that for which it was originally intended [...]. (ebd.)

Hoopers Metakritik, so relevant und bereichernd sie ist, scheint an diesem Punkt doch mehr Fragen zu motivieren als zu klären: Auf wessen Rezeption und Affekt sollen sich denn die Analytiker*innen beziehen? Weshalb soll es problematisch sein, Musikstücke zu analysieren, um musiktheoretische Instrumente und Theorien zu erklären oder zu überprüfen? Und noch gravierender: Was ist der sursprünglich intendierter Sinn eines Musikstückes? Gibt es so etwas nicht erst, wenn man die sIntentionen einer Autorin / eines Autors oder die hermeti-

12 Kriterien der Angemessenheit in der Analyse populärer Musik sind auch nicht bei den wenigen anderen Wissenschaftler*innen wie Middleton (1990, 240 f.) oder Wicke (2003) zu finden, die sie diskutiert haben. 
sche und statische Einheitlichkeit einer Musikpraxis postuliert? ${ }^{13}$

Man merkt spätestens hier, wie schwerwiegend die Fragen sind, die eine ernste epistemologische und methodologische Kritik musikalischer Analyse zutage führt - Fragen, die mit dem Hinweis auf die die Analyse leitende Fragestellung bestenfalls pragmatisch beiseitegelegt, aber nicht hinreichend beantwortet werden. ${ }^{14}$ Für simmanente Analysen<, die legitim als Mittel zur Erprobung und Entwicklung musikanalytischer Instrumente ausgerichtet werden, ${ }^{15}$ verliert der 冫Kontext $\iota$ und damit die Frage nach der Angemessenheit an Relevanz. Für Analysen aber, die Text und Kontext vermitteln wollen, gibt es eine Quelle für Kriterien der Angemessenheit, die Hooper freilich nicht erwähnt: Geschichtsarbeit. Und zwar als historische Rekonstruktion einer normativ verfassten Musikpraxis, die u. a. ästhetische Kriterien bereitstellt: wann und für wen welche Musik wie praktiziert und wertgeschätzt wurde. $^{16}$ Damit ist die musikalische Analyse auf historische Arbeit angewiesen und durch sie in ihren Wissensansprüchen begrenzt. Gleichwohl bleibt aber die Analyse auf musiktheoretisches Wissen angewiesen, denn es ist zentral, sich mit musiktheoretischen Instrumenten und Fachvokabular auszukennen, will man nicht über das Ziel hinausschießen - wie Ralf von Appen gegen kulturtheoretisch ambitionierte, aber musiktheoretisch unplausible Analysen zutreffend einwendet. ${ }^{17}$ Eine entfaltete Theorie ästhetischer Normativität populärer Musik bleibt noch ein Desideratum.

13 Beides sind Aspekte, die nicht für populäre Musik - und womöglich für keine Musik - gelten und an Probleme der musikalischen Hermeneutik eines Hermann Kretzschmars erinnern, die trotz starker Kritik heute noch kursiert, vgl. Floros 2019.

14 Hans Heinrich Eggebrecht (1979) war noch der Überzeugung, dass die regulierende Relation zwischen analytischer Fragestellung und analytischem Ansatz die Angemessenheit der Analyse garantiert.

15 Vgl. Dahlhaus 1970, 17.

16 Diese Geschichtsarbeit schließt je nach Fragestellung und Gegenstand selbstverständlich Kultur- und Mediengeschichte ein.

17 Vgl. Appen 2019, 74.
Im gleichen Maße reflektiert wie die vorherigen Kapitel beginnt Allan F. Moores Beitrag "Listening to the Sounds Music Makes" (4557). Dass Moore vor jeglicher Analyse folgende Frage stellt, zeugt hiervon: »I question whether we seriously asked swhy do I want to understand the structure of this piece? simply employing, or developing, our methodology." (45) Bemerkenswert ist außerdem Moores Relativierung des istrukturellen Verstehens von Songs, das ja als seine Hauptdevise in der Erforschung populärer Musik bekannt ist. Der Beitrag verliert aber an Schärfe, wenn Moore die Analyse populärer Musik gegen Kritiker*innen mit dem Argument verteidigt, dass Musikanalytiker*innen sanalytische Wahrheiten ‘ formulieren, während ihre Kritiker*innen sich auf erfahrungsbezogene, ssynthetische Wahrheiten beziehen. Trotz dieser wenig einleuchtenden Unterscheidung ${ }^{18}$ zieht Moore sinnvolle Konsequenzen daraus: $»$ It is this misrepresentation (as I would have it) which concerns me, and is the cause of my claim that we should be much more specific about what our analytical techniques are and what they allow us to do. (47) Dennoch postuliert Moore den analytischen Standpunkt als einfach gegeben und unmittelbar erfahrbar, wodurch hier - anders als bei Ford und Hooper - eine genuine epistemologische und methodologische Reflexion über musikanalytische Instrumente verhindert wird. Die anschließenden kurzen Analysen vieler Songs von Melanie Safka (49-53) sollen etwas über >Authentizität und 'Personas erklären. Inwiefern diese Themen mit Moores Diskussion der Analyse populärer Musik zusammenhängen, bleibt freilich unverständlich.

Eine Typologie selbstreferentieller (selfreferential) Songs erarbeiten Bethany Lowe und Freya Jarman in ihrem Beitrag "Analyse This. Types and Tactics of Self-Referential Songs" (58-76). Hier wird ein Bestand von Songs mit verschiedenen - teilweise irreführenden - Kategorien versehen und entlang der Referenzen zu ihrer eigenen Melodie, Harmonie, Instrumentation usw. typologisiert. Dass iSelbstreferenzialitäts in diesem Beitrag meistens als die

18 Der locus classicus der massiven philosophischen Kritik an dieser Unterscheidung findet sich bei Quine 1951. 
sprachliche Thematisierung straditioneller musikalischer Parameter im Songtext aufgefasst wird, scheint uns weniger interessant als eine Selbstreferenzialität im Medium Musik bzw. Klang selbst. Diese wäre allerdings mit anderen Mitteln zu untersuchen als mit einer Typologisierung dieser Art. Insofern ist die sMedienreflexivität ‘ ${ }^{19}$ populärer Musik, die die Autor*innen mit ihrem Ansatz offenzulegen versuchen, womöglich nicht nur auf dieser Ebene zu analysieren. Zudem leidet der Beitrag daran, dass der Bestand von 150 (!) Songs auf wenigen Seiten durchtypisiert wird. Es wäre sicherlich ertragreicher und übersichtlicher gewesen, sich wenige Songs vorzunehmen und tiefer in die Analyse zu gehen, wie die Autor*innen es tatsächlich bei Leonard Cohens und Jeff Buckleys Hallelujah tun.

Stan Hawkins und Jon Mikkel Broch Ålvik legen eine reichhaltige und fokussierte Analyse des Hitsongs Take On Me (1985) von A-Ha vor (77-94). Die Autoren bringen Melodie, Gesangstechnik und -einsatz in das Zentrum der Analyse, um folgende Frage zu beantworten: Was macht diesen Song so zeitlos und erfolgreich? Für ihre Analyse setzen die Autoren Notenbeispiele ein (Transkription der Synthesizer-Eingangsfanfare sowie der Gesangsmelodie im Refrain), zwei Tabellen für die Struktur der Refrain-Gesangsmelodie und des Songaufbaus sowie zwei weitere Grafiken zur Veranschaulichung rhythmischer Akzentsetzungen. Neben der melodisch prägnanten Synthesizer-Fanfare, ist es die Gesangsmelodie, der die Autoren besondere Bedeutung beimessen. Die gesangstechnisch anspruchsvollen Melodien, welche von Bariton- bis zum flötenartigen Falsettgesang reichen, geben dem Song eine melodische Vielseitigkeit, die zudem mit den damaligen Normen männlicher Stimmen brechen (78). Passend verweisen die Autoren auf Susan McClarys Begriff der soprano masculinity. (ebd.) Überzeugend formulieren Hawkins und Ålvik, dass der Einsatz von nicht korrektem Englisch - »anti-lyrics» (84) - bewusst eingesetzt werden kann, um die Aufmerksamkeit nicht auf das, was gesagt wird, sondern darauf, wie es gesagt bzw. gesungen wird, zu lenken. Lyrics können, so die Autoren, im Sinne der melodischen Gestaltung eingesetzt werden.
Dazu heißt es: »the verbal space of sTake On $\mathrm{Me}$ < is less crowded, and arguably less dependent on any meaning in the lyrics [...]: rather, the words function more as stepping stones for Harket [Morten Harket, Sänger von A-Ha] to build up the vocal melody. «(85)

Hawkins und Ålvik resümieren, dass durch eine Vielzahl an analytischen Methoden neues Licht auf Take On Me geworfen werden könne. Die prominente Stimme des Sängers, die prägnante Gesangsmelodie und die SynthesizerFanfare machen aus Take On Me ein kommunikatives Ereignis - »communcative event" (90). Der Song ist durchaus ein kommunikatives Ereignis, mehr noch: er ist ein einzigartiges klingendes und produziertes Ereignis. Hier schwächelt die Analyse von Hawkins und Ålvik, denn sie verpassen es, den Song als sproduzierte Musikı zu analysieren. ${ }^{20}$ Dass die Autoren in Take On Me die Melodiegestaltung in Gesang und Synthesizer als wichtiges Merkmal hervorheben, ist aus einer musiktheoretischen Perspektive lobenswert, verkennt jedoch die produktionstechnologische Beschaffenheit des Songs. Dessen prägnanter sKultsound ${ }^{21}$ steht emblematisch für den Einzug neuartiger, digitaler Produktionstechniken in populäre Musik, die sich in Take On Me destillieren. Es gilt also, den Song als produziertes, klangliches Objekt in seiner Eigenkomplexität und inneren Dichte zu erfassen und dabei das spezifische Verhältnis nach außen zu beschreiben. Diesem Anspruch werden Hawkins und Ålvik unserer Meinung nach nicht zur Gänze gerecht.

Im letzten Kapitel dieses Abschnittes (95114) bedient sich Lori Burns für eine transmediale und multimodale Analyse von Steven Wilsons Konzeptalbum The Raven That Refu-

20 Dabei ist es, wie Jens Gerrit Papenburg anmerkt, vielleicht gerade das, was populäre $\mathrm{Mu}$ sik als solche auszeichnet. In Bezug auf Daniel Martin Feige heißt es bei ihm, dass sich eine Ontologie der Popmusik ex negativo von einer aufs Werk setzenden Ontologie der europäischen Kunstmusik sowie von einer Performance-orientierten Ontologie der Jazzmusik abzugrenzen vermag, indem populäre Musik »primär produzierte Musik« sei. $(2019,69)$

21 Der Begriff wird hier nach Immanuel Brockhaus (2017) verwendet. 
sed to Sing (2013) beim Narratologen David Herman. Dessen Erzähltheorie strukturiert das Narrativ in vier Elemente, die Burns auf die Analyse des Konzeptalbums anzuwenden versucht: „discoursive contexts, event sequencing, worldmaking/disruption, and subjective experiences [...].« (97) Die Autorin versteht das Konzeptalbum als ein transmediales Objekt, dessen individuelle Materialitäten ızusammenarbeiten ‘, um ein Erfassen des ıgroßen Werkes` zu ermöglichen. Burns wählt eine erzähltheoretisch informierte Perspektive auf das Objekt 'Konzeptalbumı, um sich der Textualität der einzelnen Medienformen anzunehmen, was sie veranlasst, das Material als sparatextuell $\mathrm{zu}$ beschreiben (96). Damit unterscheidet sich der Ansatz von Burns grundlegend von medienund kulturwissenschaftlich informierten Blicken auf das Konzeptalbum. ${ }^{22}$ Interessant erscheint uns die Übersetzung der narratologischen Theorie, der vier Elemente Hermans, auf die musikalische Analyse.

Als >discoursive context beschreibt Burns das musikalische Genre, genauer gesagt »the sonic space that provides a setting for the musical story to unfold [...].« (101) Das Konzeptalbum wird von Burns dezidiert als produzierte Musik verstanden (»sensitive production«, 102) und im Diskurs musikalischer Genres verortet. Was Herman event sequencing nennt, definiert Burns für ihre Analyse als formale Struktur und Ordnung des musikalischen Materials in Form des Notentextes, den sie zusätzlich mit dem diskursiven Kontext, den Produktionsprozessen, zusammenbringt. Das Begriffspaar worldmaking/disrupting wendet die Autorin auf das Sound Design an. Durch produzierten Klang, so die Autorin, können Narrative geschaffen (worldmaking) und wieder erschüttert (disrupt) werden. Letztere sind als spezifische Momente, als >Brüche` oder >Emphasen ‘ im Sound Design und/oder der formalen Struktur erkennbar. Als letztes Element der Narratologie bestimmt Burns die ssubjective experiences`, die sie in Bezug auf ihr Beispiel im vokalen Ausdruck und im instrumentalen Gestus verortet. Die Vierteilung der Erzählstruktur wendet Burns zusätzlich auf die weiteren involvierten Medien an (Kurzgeschichte, Video, Lyrics) und bringt diese in einen Dialog. Schlüssig setzt die Auto-

22 Vgl. etwa Willis 1978 oder Papenburg 2017. rin ihren paratextuellen und narratologisch interessierten Anspruch am Konzeptalbum von Steven Wilson um und ermöglicht einen intensiven Umgang sowie eine dichte Beschreibung der unterschiedlichen Materialien.

\section{DIMENSIONEN POPULÄRER MUSIK IN DER ANALYSE}

\section{Timbre/Technologie}

Kevin und Brad Osborn bieten einen Ansatz an, der interessante Einblicke in die musikpraktische und studiotechnologische Produktion von Timbre gibt (133-143). Für ihre Analyse von Tools Grammy-gekröntem Album Anima (1996) führen die Autoren ein Interview mit dem Produzenten und Toningenieur des Albums David Bottrill. Neben der Beschreibung des benutzten Equipments und ausgefeilter Aufnahmetechniken erkennt Bottrill an, dass die klangliche Signatur des Albums wie eine Momentaufnahme der Band ist. Raum, Zeit, Intersubjektivität, das individuelle Talent der einzelnen Musiker, das verwendete Equipment - in dieser Versuchsanordnung wird das destilliert, was am Ende als 'kulturalisierter Schall ${ }^{23}$ hörbar wird. Auch wenn die Beschreibung der Produktionsprozesse des Albums durchaus nachvollziehbar ist und zudem durch eine Analyse der einzelnen Instrumentengruppen (Equipment, Timbre, Spieltechnik) ergänzt wird, fehlt dem Beitrag von Osborn und Osborn jegliche Innovation. Bereits 2012 hat Simon Frith ausführlich über die Rolle des Produzenten bei Rock-Produktionen berichtet. ${ }^{24}$ Nichtsdestotrotz bietet ein eingehendes Interview mit dem Produzenten, den Frith als weiteres Bandmitglied und kreative Autorität definiert, ${ }^{25}$ tiefe Einblicke in die technologische Beschaffenheit von Timbre. Das macht den Beitrag vielleicht nicht zu einer detaillierten Analyse, dafür aber informativ und aufschlussreich. Leider nutzen die Autoren das ihnen zur Verfügung stehende Demo-Material nicht vollständig aus, um ihr Verständnis von sproduziertem Timbre ‘ klarer zu formulieren. Ein direkter analytischer Vergleich zwischen den Demo-

23 Vgl. Wicke 2008, 3.

24 Vgl. Frith 2012.

25 Vgl. ebd. 
Songs und den ausproduzierten Songs des Albums wäre sicher interessant und hätte einem auf Studiotechnologie basierenden TimbreBegriff mehr Gewicht verliehen.

Victor Szabo widmet sich in seinem Beitrag der von Keith Fullerton Whitman gestellten Frage: "What Music isn't ambient in the 21st century?« (144-158) Dieser durchaus relevanten und komplexen Frage nähert sich der Autor mit einer Herangehensweise an Analyse an, die er als »design-oriented « (144) beschreibt. Dass der Design-Begriff in Auseinandersetzung mit Kulturtheorie und Sound durchaus fruchtbar und anwendbar ist, belegen jüngere Publikationen $^{26}$ sowie Curricula an einigen Hochschulen und Universitäten. ${ }^{27}$ Szabo beschreibt Design als die ästhetische Präsentation von massengefertigten Objekten, die zwischen den mutmaßlich getrennten Kategorien ıHandwerk und 'Kunst` vermitteln kann, indem beide Kategorien durch die innere wie äußere Form und die Präsentation des Objektes bedient werden. Nicht unberechtigt bemängelt der Autor, dass der Design-Begriff von der Musikwissenschaft nur stiefmütterlich angewendet wird. Dabei kann das Verständnis von recorded music, hier bezieht sich Szabo auf Adam Krims, durch den Design-Begriff eine nützliche Erweiterung erfahren, denn dieser kombiniert den ästhetischen und utilitaristischen Wert von Musik unter dem Einfluss der Ökonomie (145). Sound Designs sind dem Autor zufolge klingende flexible Komplexitäten, die auf unterschiedlichen Ebenen des Konsums und als Aufmerksamkeitsmodelle aktiv werden können, sich zudem den multiplen Interpretationen und Anwendungen durch Subjekte öffnen. Das ermöglicht Musikanalytiker*innen Verbindungslinien zwischen allgemeinen und besonderen Betrachtungen zu erkennen und das Netz von alläglichen Praktiken, Ökonomie und Subjektivierungsprozessen mitzudenken. Szabo kritisiert die textzentrierte Analyse von Musik und setzt dieser seinen soziologisch, technologisch und subjektorientierten DesignBegriff entgegen. Die daran anschließenden Analysen von Brian Enos Ambient 4: On Land

26 Vgl. etwa Murray 2019; Schulze 2019.

27 Beispielsweise an der Universität Bonn, Universität der Künste Berlin, Fachhochschule Dortmund oder HAW Hamburg.
(1982) und KLFs Chill Out (1990) kommen diesem Anspruch nach und vermögen es durchaus auch, die jeweilige Eigenkomplexität der Alben zu beschreiben. Auch wenn der von Szabo vorgeschlagene Design-Begriff attraktiv und belebend ist, bleibt unklar, inwiefern sich die design-oriented analysis von einer herkömmlichen Analyse unterscheidet, die über den Text hinausdenkt und das Netz aus Produktionstechnologie, Kultur, Ökonomie und Subjektivierung zu spannen weiß.

\section{Rhythmus, Tonhöhe und Harmonie}

Das Kapitel »Pulse as Dynamic Attending. Analysing Beat Bin Metre in Neo Soul Grooves" (179-189) von Anne Danielsen stellt einen Beitrag zur Analyse mikrorhythmischer Gestaltung von Beats in zeitgenössischer populärer Musik dar. Zunächst entfaltet Danielsen einen musikpsychologisch informierten Begriff von Metrum als »the general measure against which sounding rhythms are mapped [...].« (180) Eine Auseinandersetzung mit der Interaktion zwischen Metrum - aufgefasst als normatives virtuelles Strukturierungsschema (metric grid) und tatsächlich klingenden rhythmischen Ereignissen erlaube, so Danielsen, einen differenzierten analytischen Blick auf die Gestaltung von groove-based music und der dynamischen Wahrnehmung (dynamic attending) von Beats. Ausgehend von der Theorie rhythmischer Synchronisierung (entrainment), zeigt Danielsen anhand einer Analyse des Songs 1000 Deaths (2014) von D'Angelo, dass eine dynamische Wahrnehmungsanpassung beim Hören des Tracks stattfindet und damit eine Subsumierung von mikrozeitlichen Pulsen innerhalb eines erweiterten Beats (beat bins) eintritt. Anschließend kritisiert Danielsen Theorien, die das Metrum als zu stabil auffassen, wodurch ihre dynamische rezeptive Anpassung des Metrums beim Hören verkannt wird:

The structuring capacity in the human body has been thought of in the same way, as a nondynamic relation between external music and a non-adapting isochronous timekeeper - that is, a »clock« - within the perceiver. This approach fails to account for an experience where the actual sounds remain identical, but the perceptual response to them changes. (186) 
Danielsen schließt ihren Beitrag mit einleuchtenden Gedanken über das Zusammenkommen und die Dynamik von kognitiven Konstanten und tradierten kulturellen Normierungen beim Hören von Beats ab (186 f.).

"The Aesthetics of Drone« (207-220) ist der Titel des Beitrages von Jonathan W. Bernard. Mit einigen Erwartungen an den Begriff der Ästhetik gehen wir an einen Text mit dieser Überschrift. Doch gleich im ersten Absatz heißt es: "Any essay with the word raesthetics in its title is bound to traffic to some extent in generalizations, and this one will be no exception, partly because of the looseness, even vagueness of the term.« (207) Damit werden die Leser*innen von nun an alleingelassen, denn es werden keine weiteren Auseinandersetzungen mit dem Begriff angeboten. Wie schon im Kapitel von Christopher Doll bleibt der ÄsthetikBegriff hier also konturlos und nicht greifbar genug, als dass seine Anwendung angebracht wäre. Dem Programm des Sammelbandes folgend, stellt Bernard eine Analyse von drones im Kontext der Kunstmusik dem Einsatz von drones in populärer Musik gegenüber. So lautet eine Fragestellung: "Is there a meaningful relationship between drone in art music and drone in popular music? « (214) Bernard ist mit seinem unscharfen Begriff von ` ̈̈sthetiken der Antwort dieser Frage im Grunde auf der Spur, wenn er nach Stilen und Techniken fragt, geht dem aber nicht konsequent nach. Plausibel erklärt er, dass weder Dauer, Tonhöhe, Tonhöhenschwankungen, Laustärkeschwankungen, Timbre- noch Hüllkurvenvariationen ausschlaggebend für die Definition einer drone sind. Interessant erscheinen jedoch die Entwicklungen innerhalb einer drone, die der Autor als nicht linear, sondern als »radiative" (213) beschreibt. Eine sich durch verschiedene Materialitäten hindurchbewegende Strahlung ist eine durchaus reizvolle Metapher für drones. Darüber hinaus liefert Bernard jedoch keine analytische Definition von drones und kommt weder dem Begriff der Ästhetik noch der Beantwortung seiner Fragen hinreichend nach.

\section{Form und Struktur}

Shaughn O'Donnell wendet für seinen Beitrag (291-299) den Begriff der Collage auf Pink Floyds Atom Heart Mother Suite (1970) an. Sehr aufmerksam und präzise analysiert der
Autor den 23-minütigen Song in Bezug auf die Form, die harmonische wie thematische Entwicklung. Besonders anschaulich sind die komplexen und hilfreichen Tabellen, die die Leser*innen durch den Song und die Analyse führen. Dem Autor ist es gelungen, die Theorie und das analytische Instrumentarium zur Erforschung von Kunstmusik auf Atom Heart Mother Suite anzuwenden und dabei die Besonderheiten bei der Analyse populärer Musik im Auge zu behalten. Inwiefern sich die hier beschriebene Collagetechnik jedoch von anderen im Song vorkommenden Studiopraktiken wie dem Overdubbing unterscheidet, bleibt vom Autor unerwähnt.

John Brackett bezieht in seinem Kapitel (300-314) eine recht ungewöhnliche analytische Perspektive. Um sich der Drogenthematik des Albums Blonde (2016) von Frank Ocean zu nähern, stellt der Autor das Motiv des »Marijuana High « (310), des Rauschzustands durch Cannabiskonsum, als musikanalytische Perspektive vor. Aus einer solchen ersetzt Brackett Begriffe wie musikalische Zeit durch "Cannabis Time« (302), die durch starke Temposchwankungen, Pitch-Shifting und 'Warble-Sounds auffällt. Die labyrinthartige und verworrene Struktur einiger Songs steht stellvertretend für ein "Marijuana Thinking" (307), welches die Hörer*innen in einen Zustand der Ziellosigkeit (goallessness) und Desorientierung (disorienting experience) versetzen kann. Leider gilt dieser Zustand auch für die von Brackett vorgestellte Analyse.

Mit "Form and Time in Trout Mask Replica " (315-332) legt Peter Silberman eine ambitionierte Analyse des Albums Trout Mask Replica (1969) von Captain Beefheart \& His Magic Band vor. Mithilfe von Jonathan Kramers Ausführungen zur Momentform verschafft sich der Autor einen theoretischen Zugang zur Analyse von Beefhearts komplexem Album. Augen- und Ohrenmerk gelten den Faktoren Zeit und Form in drei ausgewählten Songs. In Feinarbeit extrahiert der Autor Momente, die er überzeugend in Notentext und Tabellen zu veranschaulichen weiß. Inwiefern sich die hier vorgestellte Kompositionstechnik der Momentform und deren analytischer Zugriff tatsächlich auf Rockund Popmusik anwenden lässt, ist jedoch fragwürdig, was auch der Autor selbst erkennt. Denn die drei analysierten Songs »have more in 
common with those of avant-garde composers of its [their] era than with contemporaneous rock or pop.« (327)

\section{Kritische Ansätze}

Der fünfte und letzte Abschnitt »Critical Frameworks: Analytical, Formal, Structural, and Political « (333-426) geht auf theoretische Fragen zurück, die in den vorigen Abschnitten aufgeworfen wurden. Dabei wird aber ein skritischer Ansatz anvisiert, weshalb sich dieser Abschnitt die schwierige Aufgabe setzt, disziplinäre Grenzen aufzuheben, um Wege für eine interdisziplinäre und kulturkritische musikalische Analyse auszuloten - sowohl für die Erforschung populärer Musik als auch für die der Kunstmusik im 20. und 21. Jahrhundert. Eine lapidare Kritik an den kulturpolitischen Forderungen nach >Grenzüberschreitung und deren künstlerischen Realisierung in der zeitgenössischen Kunstmusik der USA übt Marianna Ritchey in ihrem Beitrag »New Music in a Borderless World«(335-346). Im Fokus ihrer Kritik stehen die Werke von sogenannten sindie classical Künstler*innen, die durch den Einbezug von Instrumenten und Verfahrensweisen populärer Musik, so die Autorin, irrtümlicherweise als besonders grenzüberschreitend und avanciert zelebriert worden seien. Ritchey führt die marktorientierte Norm des ,boundarycrossing، auf eine neoliberalistische Logik zurück, die jegliche Lebensbereiche kolonisiert und jegliche Grenzen aufhebt, damit Kapital optimal fließt. Nun scheint diese Art von Kritik problematisch für populäre Musik zu sein, deren Möglichkeitsbedingungen bekanntlich marktwirtschaftlich und industriell sind - woraus aber freilich nicht folgt, dass sie keinen ästhetischen Wert erlangen kann. ${ }^{28}$

In ähnlicher kulturkritischer Perspektive adressiert der Beitrag von Eric Smialek und Méi-Ra St-Laurent (378-399) die Frage nach den Beziehungen zwischen sozialen Klassen und der Metal-Fankultur. Anhand des Notenmaterials ausgewählter Bands belegen die Autoren, dass soziale Privilegien, wie musikschulische Ausbildung oder ein Instrumentenstudium, eine » swhite-colları" (394) Metal-Ästhetik hervorbringen, die mehr mit symphonischen, moder- nen oder surrealen Kompositionen als mit populären Musikformen gemein hat.

Michael Spitzer wendet in seinem Beitrag (400-415) die sPostmemory Theory< von Marianne Hirsch auf das Album In the Aeroplane over the Sea (1998) von Neutral Milk Hotel an, um Diskurse zum kulturellen Gedächtnis, kollektivem Trauma und Erinnerungskultur zu aktivieren, wohingegen sich Christa Anne Bentley für ihr Kapitel (416-426) mit dem Diskurs um die Singer-Songwriter-Bewegung der späten 1960er und frühen 1970er Jahre auseinandersetzt. Bentley stellt heraus, dass während des Folk-Revivals Verbindungen zu westlicher Kunstmusik und Literatur Praktiken des Songwritings maßgeblich verändert haben und ferner dazu geführt haben, dass aus marketingstrategischen Gründen Kategorisierung der Künstler*innen als scomposer (421), spoet`(419) oder snovelist (420) vorgenommen wurden. Auch wenn einige der Beiträge im letzten Abschnitt des Sammelbandes ihre Qualitäten aufweisen und ihre Gedanken anregend sind, stellt sich uns die Frage, ob hier tatsächlich neue und erweiterte kulturkritische Perspektiven präsentiert werden, die nicht bereits in den PMS ausführlich erprobt und diskutiert wurden.

Nach der Lektüre des Bandes scheint es uns, als führe die theoretische und methodologische Auseinandersetzung mit zeitgenössischer Kunstmusik nur punktuell zur Etablierung und Erweiterung von Ansätzen zur Analyse populärer Musik. Denn die diskutierten Beiträge beziehen sich kaum - in der Regel gar nicht - auf zeitgenössische Kunstmusik oder deren (musikanalytische) Erforschung. Simon ZagorskiThomas setzt sich sogar in seinem Beitrag (117-132) von an Kunstmusik orientierter Musiktheorie emphatisch ab, um einen »alternative approach to music theory « (121) zu profilieren. Lediglich die Kapitel von Neil Newton, Peter Silberman, Shaughn O’Donnell und Ciro Scotto lösen die Ambitionen des Sammelbandes ein. Insbesondere Scottos Beitrag diskutiert ausführlich zeitgenössischen Ansätze zu pitch class und set theory, um ein neuartiges Instrument für die Analyse von Tonbeziehungen in Metalsongs zu entwickeln. Ob diese recht diffi- 
zile Herangehensweise sich tatsächlich lohnt, darf bezweifelt werden. Auch die Beiträge von Silberman und O'Donnell wissen Methoden zur Erforschung von westlicher zeitgenössischer Kunstmusik auf populäre Musik anzuwenden - jedoch nur auf solche, die ohnehin avantgardistischen Charakter aufzeigt und damit zwischen den Stühlen steht. Inwiefern die vorgestellten Analyseinstrumente also auch für andere Formen populärer Musik praktikabel sind, bleibt ungewiss. Für weitere Dimensionen populärer Musik bedienen sich die Autor*innen des vorliegenden Bandes Theorien und Methoden, die entweder gezielt zur Erforschung populärer Musik entworfen wurden oder aus anderen Disziplinen, Feldern und Studies herkommen. Das gilt beispielsweise für Danielsen, deren führende Theorien und Instrumente zur Analyse von Mikrotiming aus ihrer Auseinandersetzung mit Musikpsychologie und populärer Musik hervorgegangen sind. Ähnliches gilt für andere Beiträge etwa die von Nicole Biamonte (190-206) und John Covach (277-290), die den analytischen Fokus auf die die formbildende Funktion (phrases, sections und section groupings) von diversen musikalischen Elementen in Rocksongs setzen. Weder Biamonte noch Covach beziehen sich auf Theorien oder Methoden zur Erforschung zeitgenössischer Kunstmusik.

Den musikanalytischen Zugang auf populäre Musik durch einen Companion zu verstärken, erscheint uns angesichts der fachpolitischen Lage der PMS begrüßenswert. Und einige Kapitel des Bandes leisten tatsächlich einen substanziellen Beitrag zur Analyse populärer Musik - bei Hoopers Beitrag handelt es sich womöglich um die scharfsinnigste epistemologische Diskussion zur Analyse populärer Musik seit Richard Middletons kanonischem Studying Popular Music. ${ }^{29}$ Dennoch kann das Konzept des Bandes hinsichtlich einer Erfüllung seiner selbstgesetzten Ansprüche nicht restlos überzeugen. Diesbezüglich bleiben aber noch weitere Fragen im Raum stehen: Warum sollte sich gerade die Analyse populärer Musik an den Theorien und Methoden zur Erforschung zeitgenössischer Kunstmusik orientieren? Lassen sich nicht vielleicht auch umgekehrt neue Fragen an die Kunstmusik des 20. und 21. Jahrhunderts stellen? Um diesen Fragen nachzugehen, hätte man die Abschnitte zu Timbre und Technologie sowie zu kritischen Ansätzen breiter besetzen müssen, sodass Begriffe wie 'Kultsounds`, 'Musikformulares, 'Presets`, 'produzierte Musikı oder sphonographische Arbeits ausführlich hätten behandelt werden können. Mehr noch: Man hätte sich ein deutlich breiteres Repertoire an populärer Musik vornehmen müssen. Davon hätte nicht nur die Erforschung zeitgenössischer Kunstmusik profitieren können. Vielmehr hätte man auch viele genuin musikanalytische Herausforderungen - gemäß ihren eigenen ästhetischen Standards und gemäß ihrer höchst artifiziellen Klanglichkeit angehen können; Herausforderungen, die viele Phänomene der populären Musik (seien es übersteuerte Rock ' $n$ ' Roll-Aufnahmen der 1950er Jahre, Beatboxen, DJ-Sets, Musik als Stream oder spersonalisierter Klang) noch heute stellen.

Max Alt, José Gálvez

\section{Literatur}

Appen, Ralf von/ André Doehring (2014), »Analyse populärer Musik. Madonnas Hung Up«, in: Populäre Musik. Geschichte - Kontexte - Forschungsperspektiven, hg. von Ralf von Appen, Nils Grosch und Martin Pfleide-

Appen, Ralf von / André Doehring / Dietrich Helms / Allan F. Moore (Hg.) (2015), Song Interpretation in 21st-Century Pop Music, Farnham: Ashgate. 
Appen, Ralf von (2019), „Populäre Musik zwischen musikwissenschaftlicher Analyse und kulturwissenschaftlicher Deutung", Musik \& Ästhetik 23/2, H. 90, 71-75.

Bertram, Georg W. (2014), Kunst als menschliche Praxis. Eine Ästhetik, Berlin: Suhrkamp.

Brockhaus, Immanuel (2017), Kultsounds. Die prägendsten Klänge der Popmusik 19602014, Bielefeld: transcript.

Brøvig-Hanssen, Ragnhild/ Anne Danielsen (2016), Digital Signatures. The Impact of Digitization on Popular Music Sound, Cambridge, MA: MIT Press.

Butler, Mark J. (2006), Unlocking the Groove. Rhythm, Meter, and Musical Design in Electronic Dance Music, Bloomington: Indiana University Press.

Chaker, Sarah / Jakob Schermann / Nikolaus Urbanek (Hg.) (2018), Analyzing Black Metal Transdisziplinäre Annäherungen an ein düsteres Phänomen der Musikkultur, Bielefeld: transcript.

Dahlhaus, Carl (2001), »Analyse und Werturteil" [1970], in: Allgemeine Theorie der Musik II (= Gesammelte Schriften in zehn Bänden, Bd. 2), hg. von Hermann Danuser in Verbindung mit Hans-Joachim Hinrichsen und Tobias Plebuch, Laaber: Laaber, 11-76.

Doehring, André (2012), »Probleme, Aufgaben und Ziele der Analyse populärer Musik«, in: Black Box Pop. Analysen populärer Musik, hg. von Dietrich Helms und Thomas Phleps, Bielefeld: transcript.

Eggebrecht, Hans Heinrich (1979), "Zur Methode der musikalischen Analyse«, in: ders., Sinn und Gehalt. Aufsätze zur musikalischen Analyse, Wilhelmshaven: Heinrichshofen, 7-42.

Fink, Robert / Melinda Latour / Zachary Wallmark (Hg.) (2018), The Relentless Pursuit of Tone. Timbre in Popular Music, New York: Oxford University Press.

Floros, Constantin (2019), »Musikalische Hermeneutik«, in: Historische Musikwissenschaft. Gegenstand - Geschichte - Methodik, hg. von Frank Hentschel, Laaber: Laaber, 256-272.

Frith, Simon (2012), »The Place of the Producer in the Discourse of Rock", in: The Art of
Record Production. An Introductory Reader for a New Academic Field, hg. von Simon Frith und Simon Zagorski-Thomas, Farnham: Ashgate, 207-222.

Gayraud, Agnès (2019), Dialectic of Pop, Falmouth: Urbanomic.

Hennion, Antoine (2015), The Passion for Music. A Sociology of Mediation, Farnham: Ashgate.

Janz, Tobias (2012), „Zur Konstruktion musikhistorischen Wissens in der musikalischen Analyse«, in: Konstruktivität von Musikgeschichtsschreibung. Zur Formation musikbezogenen Wissens, hg. von Sandra Danielczyk, Christoph Dennerlein, Sylvia Freydank, Ina Knoth, Mathias Maschat, Lilli Mittner, Karina Seefeldt und Lisbeth Suhrcke, Hildesheim: Olms, 71-91.

Mersch, Dieter (2010), Posthermeneutik, Berlin: Akademie.

Middleton, Richard (1990), Studying Popular Music, Buckingham: Open University Press.

Moore, Allan F. (1993), Rock: The Primary Text. Developing a Musicology of Rock, Buckingham: Open University Press.

Müller, L. J. (2018), Sound und Sexismus. Geschlecht im Klang populärer Musik. Eine feministisch-musiktheoretische Annäherung, Hamburg: Marta Press.

Murray, Leo (2019), Sound Design Theory and Practice. Working with Sound, Abingdon: Routledge.

Osborn, Brad (2016), Everything in its Right Place. Analyzing Radiohead, New York: Oxford University Press.

Palfy, Cora S. (2016), »Human After All: Understanding Negotiations of Artistic Identity through the Music of Daft Punk«, in: The Oxford Handbook of Music and Virtuality, hg. von Sheila Whiteley und Shara Rambarran, New York: Oxford University Press, 282-305.

Papenburg, Jens Gerrit (2017), »Konzeptalben als 'große Werke` populärer Musik?«, Musik \& Ästhetik 21/4, 30-45.

Papenburg, Jens Gerrit (2019), »Popmusik als sproduzierte، Musik", Musik \& Ästhetik 23/2, H. 90, 68-71. 
Quine, Willard Van Orman (1951), „Two Dogmas of Empiricism«, The Philosophical Review 60, 20-43.

Rehding, Alexander (2006), „On Libraries, Encyclopedias and Contemporary Theorizing «, Contemporary Music Review 25/3, 205-209.

Schulze, Holger (2019), Sound Works. A Cultural Theory of Sound Design, New York: Bloomsbury.

Sloan, Nate / Charlie Harding (2020), Switched On Pop. How Popular Music Works, and Why it Matters, New York: Oxford University Press.

Tagg, Philipp (2015), Music's Meanings. A Modern Musicology for Non-Musos, New York: The Mass Media Music Scholars' Press.
Wicke, Peter (1992), » Populäre Musikı als theoretisches Konzept", in: PopScriptum. Beiträge zur populären Musik 1, 6-42. https://edoc.hu-berlin.de/bitstream/handle/ 18452/20904/pst01_wicke.pdf (21.4.2020)

Wicke, Peter (2003), „Popmusik in der Analyse«, Acta Musicologica 65/1, 107-126.

Wicke, Peter (2008), „Das Sonische in der Musik«, in: PopScriptum 10, 1-21. https://edoc.huberlin.de/bitstream/handle/18452/21050/pst 10_wicke.pdf (21.4.2020)

Willis, Paul E. (1978), Profane Culture, London: Routledge \& Kegan Paul.

Alt, Max / Gálvez, José (2020): Ciro Scotto / Kenneth Smith / John Brackett (Hg.), The Routledge Companion to Popular Music Analysis. Expanding Approaches, New York: Routledge 2019. ZGMTH 17/1, 193-205. https://doi.org/10.31751/1033

(C) 2020 Max Alt (maxalt@uni-bonn.de), José Gálvez (galvez@uni-bonn.de) Rheinische Friedrich-Wilhelms-Universität Bonn [University of Bonn], Rheinische Friedrich-WilhelmsUniversität Bonn [University of Bonn]

Dieser Text erscheint im Open Access und ist lizenziert unter einer Creative Commons Namensnennung 4.0 International Lizenz.

This is an open access article licensed under a

Creative Commons Attribution 4.0 International License.

eingereicht / submitted: 09/04/2020

angenommen / accepted: 09/04/2020

veröffentlicht / first published: 15/06/2020

zuletzt geändert / last updated: 15/06/2020 